\title{
Strategic changes in task performance in simulated car driving as an adaptive response to task demands
}

\author{
Fokie Cnossen *, Talib Rothengatter, Theo Meijman \\ Department of Psychology, University of Groningen, Gr. Kruisstraat 2/1, 9712 TS Groningen, Netherlands \\ Received 6 February 2000; received in revised form 8 June 2000; accepted 15 September 2000
}

\begin{abstract}
Car drivers appear to reduce their driving speed in high task demand situations. Summala's [Safety Sci. 22 (1996) 103-117]; [in: J.A. Rothengatter, \& E. Carbonell Vaya (Eds.), Traffic and Transport Psychology: Theory and Application, Pergamon, Oxford, 1997, pp. 41-52] model of behavioural adaptation (MBA) also assumes that drivers increase speed in low task demand situations or attend to additional tasks more. The present study investigated the relation between driving speed and task demands in simulated driving. Participants were observed under three speed conditions, driving fast, driving as if taking a driving test, and following a fast-driving car. The same route was driven twice under each of these speed conditions: once with and once without the concurrent performance of an auditory short-term memory task. All other things being equal, driving fast required more effort than driving more slowly, which was not compensated for by better memory performance. This refutes one assumption of the MBA. When following a fast-driving car, participants invested less effort than when driving fast. As auditory route guidance messages were embedded within the memory task, participants were forced to attend the memory task in all rides of the Fast and Accurate conditions, but not in the Car Following conditions. This can also explain why the memory task had no effect on cognitive effort. It is concluded that car drivers prioritise their task goals. (C) 2001 Elsevier Science Ltd. All rights reserved.
\end{abstract}

Keywords: Driving behaviour; Task demands; Route guidance systems; Mental effort; HRV; Strategies

\section{Introduction}

It is generally assumed that operators in complex hierarchical tasks deal actively with task demands. In order to compensate for high task demands, operators have been shown to use a

\footnotetext{
${ }^{*}$ Corresponding author.

E-mail address: fokiacob@inn.nl (F. Cnossen).
} 
number of strategies (Bainbridge, 1974; Hockey, 1993, 1997). One strategy is to invest more effort in the task. Another strategy is to adopt less demanding working strategies, which involve fewer manipulations of information or less use of working memory (for example, writing things down instead of trying to remember them; resorting to well-practised routines instead of working out the best solution) or adopt a more relaxed method of working (working more slowly or less accurately). A third strategy is to skip subsidiary tasks that are not essential for achieving the main task goal. The effect of this strategy is an increased focus on the main task, at the expense of subsidiary tasks (see also Hockey, Wastell, \& Sauer, 1998). Thus, the compensatory control model of Hockey (1997) assumes that overall task performance may decrease when less demanding strategies are used or subsidiary tasks are skipped, but that the primary task goal is usually protected against degradation.

An example of a complex dynamic task is car driving, and though traffic behaviour research has not always explicitly focussed on adaptive strategies of car drivers, already in the 1960s the driving task was described as a self-paced task (Taylor, 1964), where drivers change their behaviour in response to secondary task demands. For example, Brown and Poulton (1961) and Brown (1962) showed that performance on a secondary auditory task decreased when demands of the primary driving task increased. Later studies confirmed these results and found evidence for a number of strategies in dealing with high task demands, most notably a change in driving behaviour and neglect of a subsidiary task. For example, Harms (1991) found evidence for both these strategies: drivers decreased their driving speed and were slower in responding to a mental arithmetic task in more demanding traffic situations.

Other studies have also shown that car drivers adopt less demanding strategies by changing their driving behaviour when task demands increase. It has been found that car drivers reduced their driving speed when task demands increased (e.g., Cnossen, Meijman, \& Rothengatter, 2000; Dingus et al., 1997; Pohlmann \& Traenkle, 1994), while Noy (1989) showed that drivers increased their headway when performing an additional task, involving monitoring a display. Car drivers also neglect subsidiary activities when task demands increase. Various studies have, for example, found that participants tend to check their rear-view mirror less with increased task demands, for example, when driving with a visual route guidance system or when performing additional memory tasks (Landsdown, 1997; Fairclough, Ashby, \& Parkes, 1993; Brookhuis, De Vries, \& De Waard, 1991). In all these studies, mirror checking was not an important subtask. Landsdown (1997) performed his study in relatively low traffic densities and in such circumstances, drivers may have had enough time to check on other traffic. Fairclough et al. (1993) draw attention to the adaptive character of their participants' behaviour, noting that different traffic environments and traffic densities resulted in different visual scanning patterns. In the study by Brookhuis et al. (1991), drivers were not allowed to overtake or change lane, which largely reduced the need to check the mirrors. This suggests that with increased demands, drivers chose to neglect actions that were not essential in the given circumstances. De Waard (1991) indeed showed that in situations where task demands were high and checking the mirrors was important (driving on a combined entrance/exit section of a motorway), drivers did not decrease the frequency with which they checked their mirrors. Instead, they decreased the total glance duration on traffic irrelevant objects. Indeed, Crundall and Underwood (1998) have shown that experienced drivers' eye movements change across differing road types, adapting their looking behaviour to the circumstances. Furthermore, Crundall, Underwood, and Chapman (1999) showed that hazardous events redirect 
attention away from extra-foveal regions of the functional field of view toward the hazard at the point of fixation. These studies clearly illustrate the adaptive component in visual scanning behaviour.

An important issue is what the possible mechanisms are behind the behavioural adaptations. The 1970s saw the breakthrough of motivational models (Summula, 1985) which assumed that driving is a self-paced task and that drivers select the amount of risk they are willing to tolerate (Ranney, 1994). That is, these models assumed an active role for the driver in dealing with task demands. Two approaches were highly influential: the risk homeostasis theory (Wilde, 1982) and the zero-risk theory (Näätänen \& Summula, 1976). The risk homeostasis theory proposed that driving behaviour is aimed at maintaining a subjective target risk level, i.e., drivers adjust their speed to keep subjective risk levels constant. An implication of this theory is that drivers will not only, for example, drive slower to compensate for high task demands, but will also react to safetyenhancing improvements by driving faster or less cautiously to re-establish the target level of subjective risk. Main criticisms included a confusion between aggregated and individual levels of analysis, lack of internal mechanisms that account for the higher-order cognitive functions with which the models deal (risk, beliefs), and exclusion of cognitive mechanisms (Michon, 1985). The zero-risk model assumed that in the dynamic driving situation, drivers control safety margins rather than risk; only when subjective risk or fear increases above a certain threshold, will risk influence driving behaviour. Although the two models differ in the precise way in which risk plays a role in driving, they have in common that if subjective risk is considered too high, drivers will change their driving behaviour and will, for example, slow down. Note that the task demands do not lead to an adjustment of driving behaviour directly but only indirectly through subjective risk.

The zero-risk model already used safety margins as the control variable, and these were later also incorporated in the hierarchical model of behavioural adaptation by Summala $(1996,1997)$. Behavioural adaptation, also called risk compensation, is the phenomenon that drivers are inclined to react to changes in the traffic system, whether they be in the car, in the road environment, in road or weather conditions, or in their own skills or states, in accordance with their motives (Summala, 1997). More specifically, it may also refer to behavioural changes specifically triggered by safety measures (such as airbags, antilock brakes, or collision avoidance systems), which cancel out the intended positive safety effects of the measures. Rather than control of subjective risk, it is control of available time that is central to this model. Available time is reflected in time margins, the temporal distance to an object, as in time-to-line crossing (TLC), time headway, etc. It is assumed that time margins are the control variable for drivers. For example, for a similar TLC, 'decreasing road width or increasing curvature calls for slowing down (or more effort), and wider or straighter road allows higher speeds or more time' (Summala, 1997, p. 44). Thus, drivers try to keep available time constant, and driving speed serves an important role in doing so. In this model, mental workload and shortage of available time (or short time margins) are closely related (see also Hancock \& Caird, 1993, for a similar position on workload). In Summala's words: 'to maintain speed when the road standard (e.g., width) decreases we have to put more effort into the task, or slow down' (1996, p. 112). Borrowed from earlier risk models is 'a tendency to go to the limit, which may be postulated as an inherent motivational pressure to increase speed' (1996, p. 111). Thus, as in risk compensation models, with increasing time available (for example when task demands are low), drivers are assumed to increase speed or, alternatively, allocate more time to in-car tasks, resulting in shorter psychological time margins. 
The behavioural adaptation model by Summala $(1996,1997)$ is in accordance with the general literature on task demands, in the sense that increased task demands may lead to slower driving speeds. However, the compensatory control model of Hockey (1997) does not predict that when task demands are low, operators will increase their speed or accuracy to keep workload constant. In the general literature on complex dynamic task performance, it is assumed that fundamental to task performance is the protection of goal directed behaviour, not keeping levels of effort or task demands constant. In Summala's conception, higher-level goals do play a role in modifying lowerlevel behaviour, but the main control variable is available time or workload. This leads to different predictions for low task demand situations: the behavioural adaptation model predicts an increase in driving speed, or increased time spent on additional tasks to keep available time or workload constant, whereas the compensatory control model has no specific predictions for low task demands.

A typical example of low task demands is the reduced arousal state due to time on task. Truck drivers have been shown to increase time headway when driving in convoy at night (Fuller, 1981) whereas Van der Hulst, Meijman, and Rothengatter (2000) found a positive relation between levels of fatigue and time headway: fatigued drivers increased their time headway more than drivers who did not report an increase in fatigue. Fuller (1981) interpreted the increase in time headway as an increase in caution to compensate for reduced visibility and/or for non-optimal arousal level. Van der Hulst et al. (2000) suggest that drivers who are fatigued become less willing to invest effort into the task, and therefore increase their safety margins. This argues against the assumption of the behavioural adaptation model that workload (and thus effort) should remain constant, although it may still be argued that because fatigue drivers' reaction will be slower, psychological time margins (i.e., time margins that include slower reaction times) may still have remained constant. However, Egelund (1982) showed that after prolonged driving, heart rate variability (more specifically, the $.10 \mathrm{~Hz}$ component) increased, which he interpreted as a measure of increased fatigue; however, the $.10 \mathrm{~Hz}$ component is more generally interpreted as a measure of mental effort (e.g., Mulder \& Mulder, 1990), and Egelund's results thus showed that drivers invest less effort after prolonged driving. This argues against the assumption of the behavioural adaptation model that drivers try to keep workload levels constant.

However, not all researchers interpret reductions in driving speed as an adaptive response to high task demands. Dingus et al. (1997) interpreted the fact that drivers reduced their speed when looking at a visual route guidance display as an indication of driver 'inattention' to the driver task. Drivers slow down because they invest more effort in the secondary task and less effort in maintaining speed, just as car drivers will swerve more when they focus on an in-car device and not on the road. Many researchers stress the importance of driver 'inattention' in accidents. Drivers' attention is diverted from the driving task and focused on processing additional information or performing additional tasks. It has been argued that driver inattention plays a role in about $30-50 \%$ of accidents, and that the introduction of in-car displays could increase this number (Verwey, 1993). Another fear is that of narrowing of attention. Alm and Nilsson (1995) warned that high task demands may lead to narrowing of attention, which is unwanted in situations that ask for a more global type of attention, for example, near intersections. However, results from Crundall et al. (1999) show that attention is actually directed at hazardous events when they present themselves, away from extra-foveal regions of the visual field. 
There is thus some controversy regarding the effects of secondary tasks on driving performance and the underlying mechanisms. The differences are, however, important. If changes in driving behaviour are caused by driver 'inattention' (i.e., no attention is paid to the driving task), then these changes are unintended and unwanted. This can be considered an inadequate response of the driver to the increased demand. On the other hand, if driving behaviour is changed as an adaptive response to high task demands, the car driver is actively restructuring the task to meet the task demands. The latter would be predicted on the basis of the general literature and also by the behavioural adaptation model by Summala $(1996,1997)$ concerning the effects of high task demands on task performance. The last two models mainly differ in their predictions regarding low task demand situations.

In an earlier study (Cnossen et al., 2000), the relation between task demands and driving behaviour was investigated. Primary task demands were increased by driving in high traffic density, and by using a paper map for navigation (arriving at the destination is the main task goal in driving, which implies that map reading is a very relevant subgoal). Secondary task demands were increased by presenting a working memory task when participants were driving. Car drivers were found to reduce their driving speed only when primary task demands increased, not when performing the memory task. Increases in secondary task demands instead, resulted in skipping the subsidiary task when task demands increased beyond capability or motivation of the participants. Heart rate variability (HRV) was used as a measure of mental effort, and the result of participants' strategies was that HRV showed no increase in mental effort in this study. This suggests that car drivers are indeed adjusting their behaviour to cope with high task demands. However, due to the nature of the experimental design, a direct relation between task demands, driving speed and mental effort could not be inferred.

The present experiment tried to clarify the relation between task demands, driving speed and mental effort by having car drivers drive at different speeds and measuring their invested effort. If car drivers reduce driving speed to reduce task demands, mental effort should, all other things being equal, decrease with decreasing driving speed; otherwise, a reduction of speed cannot have the effect of reducing task demands. In the experiment, performed in the same urban environment as in the previous study, participants received three different instructions concerning driving speed. In the Accurate condition, they were instructed to drive as accurately as possible, as if taking a driving test. In the Fast condition, participants were instructed to drive as fast as possible, as if they were late and in a hurry. In the Car Following condition, participants had to follow a relatively fast driving car. This last condition was included to have direct control of participants' driving speed and thus to ensure that driving speed was high. The speed instructions, therefore, resulted in one condition in which participants drove relatively slow (Accurate) and two conditions in which participants drove relatively fast (Fast and Car Following).

Each speed condition (Fast, Accurate and Car Following) involved two rides, which differed in terms of secondary task demands. In all rides, travel news was presented to the participants. The travel news reported 30 traffic jams on Dutch motorways. In one ride under each speed condition, the participants had to the name the longest traffic queue at the end of the ride; in the other ride under each speed condition, they were not required to process the information. Consequently, six rides had to be completed, representing three speed conditions and two levels of secondary task demands. 
There were a number of different expectations. The compensatory control model predicts that, all other things being equal, driving speed and effort levels are inversely related. Thus, the Accurate condition, where driving speed is lowest, should be associated with lower effort levels than the other two speed conditions. Also, performing the memory task should increase effort levels. A weaker prediction is that in high speed conditions, high task demands may lead drivers to give up the memory task, resulting in worse memory task performance in these conditions. These effects are also predicted by the behavioural adaptation model but this model has an additional prediction: precisely because of lower driving speed in the Accurate condition, and thus lower effort levels, performance on the additional memory task should be better in that condition than in the other conditions, because psychological time margins have increased due to lower driving speed. Additionally, with available time or workload as the control variable, it predicts that effort levels should be equal for all conditions.

\section{Method}

\subsection{Participants}

Twenty participants participated in the study, 10 male and 10 female. Except for five participants, all participants had taken part in earlier experiments using the same driving simulator. Their age ranged from 24-39 (mean age 31.6). All participants had held a driving licence for more than $3 \mathrm{yr}$ (mean was $12.2 \mathrm{yr}$ ). Mean kilometres driven per annum was 15,100. Participants were paid for their participation.

\subsection{Experimental environment and conditions}

The experiment was performed in a driving simulator (Van Wolffelaar \& Van Winsum, 1995). The simulator car was a BMW 525 with original controls linked to the driving simulator. A graphical workstation (Silicon Graphics Skywriter) generates the images, which are projected on a panoramic screen with a $165^{\circ}$ angle of vision horizontally and a $45^{\circ}$ angle of vision vertically. Using the network specification facility, we built an urban environment with many intersections and buildings. There were main and secondary roads, traffic signs and traffic lights. The road network consisted mostly of straight roads but there were curved roads as well. During the experiment, other interactive traffic drove on the road network.

Participants made six rides representing three speed conditions (Fast, Accurate and Car Following) and two secondary-task demand conditions. Half of the participants started in the Fast condition, in which participants were requested to try to reach their destination fast quickly as possible, as if they were in a great hurry, and already late. The other half of the participants started in the Accurate condition, in which participants were requested to drive as accurately as possible, as if they were taking a driving test. The participants were also instructed to observe the speed limit of $50 \mathrm{~km} / \mathrm{h}$ in this condition. Note that in the Accurate condition, participants were not instructed to drive slowly, merely to drive accurately. During these rides, participants received simple auditory route guidance ( $R G$ ) messages (go right, go left) presented by a digitised female voice. If participants had to go straight ahead at an intersection, no RG message was presented. 
With the scenario specification facility of the simulator, all participants were presented with RG information at the same locations in the road network.

For all participants, the last speed condition of the experiment was the Car Following condition. In the Car Following condition, participants did not receive RG messages but were instructed to follow a car driving in front. At the first intersection of a ride, a dark blue car would arrive from the right. Participants were told that if they followed that car, they would automatically reach their destination. Participants were notified that the lead car would drive rather fast and that, for that reason, they had to be careful not to lose it in traffic. The lead car aimed to drive $60 \mathrm{~km} / \mathrm{h}$, and would not yield to traffic at intersections, of which the participants were unaware. This condition was always the last condition as it involved relatively reckless driving by the car to be followed, and we did not want participants to be tempted to copy this behaviour in the Fast condition.

All three speed conditions consisted of two rides. Task demands were varied by the inclusion of a memory task in half of the rides. During all rides, participants were presented with one broadcast of travel news; the presentation of the 30 traffic jams lasted about $120 \mathrm{~s}$ in total. In either the first or second ride of a speed condition (balanced across participants), participants were told beforehand to listen carefully to the travel news as they would be asked to name the location and length of the longest traffic jam of the list (e.g., Amsterdam, $8 \mathrm{~km}$ ) at the end of the ride. In the other conditions, travel news was presented but participants were instructed to ignore this information. In all cases, the information consisted of 30 traffic jams on 15 different existing Dutch motorways, varying in length between 3 and $9 \mathrm{~km}$. Some traffic jams had equal lengths. There could be more than one traffic jam on the same location, but there were never more than three. Traffic jams on the same location were never presented immediately after each other and never had equal lengths. If a traffic jam had the same length as the previous longest traffic jam, participants were instructed that they should remember the last traffic jam, replacing the previous longest traffic jam. In each list, the longest traffic jam was replaced seven times by a traffic jam of greater or equal length. The presentation of the traffic news was delayed when a RG message was presented and resumed after the RG message had finished.

\subsection{Procedure}

Upon arrival, the participants filled out a short questionnaire about their driving experience and health. Participants made a test drive of about $10 \mathrm{~min}$ in the urban environment of the experiment. Naive participants received extra training, until they were able to make smooth turns in the urban environment. Instructions about the experimental conditions were given in written form.

A short version of the memory task (60 s) was practised while participants were stationary; then, while driving, a short (60 s) and long (120 s) version were practised. Participants received feedback on their performance on the task. This was followed by two practice rides of $120 \mathrm{~s}$ identical to the experimental rides. In one of these rides, participants had to ignore the travel news. Participants were instructed to drive as they would normally do, and received no feedback. After this, the experiment started with either the Fast or Accurate condition (balanced across participants). The last condition for all participants was the Car Following condition. 
Reference measurements of heart rate were made during for the 3 min prior to the last two practice rides and prior to each speed condition (i.e., before Accurate, Fast and Car Following). At the end of a reference measurement, a digitised female voice requested participants to start the engine and drive off. When the participant reached a specific location in the route (about $10 \mathrm{~s}$ after starting to drive), the travel news started, and an event code was automatically generated by the driving simulator computer to mark the beginning of the condition in both the heart rate recording file and the driving parameters recording file. The end of each condition was also a specific location in the ride, and when the subject arrived there, the same electronic voice asked the subject to stop and park the car on the shoulder of the road, and an event code was automatically generated to mark the end of the condition in all recording files. Immediately after a subject had parked and come to a stop, he or she was asked to give the total length of the traffic queues (in the memory task conditions); no feedback on accuracy was given. Subjects were then requested to fill out a self-report scale for mental effort (see below). Then followed the instructions about the next condition, and when the subject was ready, the next condition was started by the auditory instruction to start the engine and drive off.

\subsection{Data collection and analysis}

The driving performance measures were speed, standard deviation of lateral position (SDLP, a measure of amount of car swerving), and standard deviation of steering wheel angle (SD Steer, a measure of steering performance). All measures were sampled at $5 \mathrm{~Hz}$. Averaged driving speed, averaged SDLP and averaged SDLP were calculated for each ride. These measures were averaged across those periods when participants were actually driving to exclude the confounding effect that waiting at traffic lights etc., would have on averaged speed; the number of such periods could vary between participants and conditions.

Self-report (Rating Scale Mental Effort, RSME, Zijlstra, 1993; Verwey \& Veltman, 1996), heart rate (HR) and HRV were used as measures of mental effort. Heart rate is a more general measure of arousal, whereas, HRV is more specifically sensitive to cognitive processes involved with information manipulation in working memory (Aasman, Mulder, \& Mulder, 1987; Wilson \& Eggemeier, 1991; Wiethof, 1997).

After completing each condition, participants were asked to indicate their invested mental effort on the RSME, a uni-dimensional scale of mental effort. This scale runs from 0 to 150 and has been shown sensitive to changes in mental effort (Zijlstra, 1993; Verwey \& Veltman, 1996). If a ride included the memory task, participants were first asked to name the length and location of the longest traffic jam before they were asked to rate their effort. This procedure prevents unnecessary decay of the contents of working memory.

Inter-beat intervals (IBI) were computed from the event file (heart rate in bpm can be calculated as $60,000 / I B I)$ and corrected for physiological and measurement artefacts. Heart rate variability in the selected frequency domain (HRV) was calculated with CARSPAN (Mulder, Van Dellen, Van der Meulen, \& Opheikens, 1988). In agreement with other research (e.g., Aasman et al., 1987; Mulder \& Mulder, 1990; Wiethof, 1997), we used the mid-frequency band .07-.14 Hz as a measure of mental effort. IBI of a condition was expressed as percentage relative to the preceding reference period. HRV values for each condition were normalised by logarithmic transformation and expressed relative to the preceding reference periods (the HRV values are thus expressed as 
$\ln ($ task $)-\ln ($ rest $)$. For the transformed data, the law of initial values is no longer valid (Van Roon, 1998) and this log transformation, therefore, allows analyses of variance on the HRV values. When mental effort increases, heart rate variability decreases.

All data were analysed with analyses of variance, except performance on the memory task. Because responses on the memory task were dichotomous (either correct or incorrect), they were analysed with McNemar tests for significance of changes. As the results for the Accurate and Fast condition most clearly test the effect of driving speed on mental effort, pair-wise comparisons were made testing for significant differences between these conditions. Also, differences between the Fast and Car Following condition were tested. There were no main effects of the order in which conditions were presented (Fast or Accurate as first condition; the memory task in the first ride in a speed condition) and order was therefore not considered in the statistical tests.

\section{Results}

Three naive participants, all female, did not complete the experiment because of simulator sickness. Heart rate measurements were completely or partially lost for three participants due to apparatus failure. Consequently, complete data were obtained for 14 participants, and data will therefore be analysed for these 14 participants ( 7 males and 7 females) only. None of the variables showed a significant effect of gender.

\subsection{Driving performance}

The speed instructions indeed resulted in different driving speeds (see Fig. 1) across speed conditions (Hotelling's $T=18.48 ; P<.000$ ). Pair-wise analyses showed that mean speed in the Fast condition was significantly higher than in the Accurate condition $(F(1,13)=15.35$; $P<.003)$, and speed in the Car Following condition was significantly higher than in the Fast condition $(F(1,13)=11.13 ; P<.006)$. There was no significant effect of the memory task on

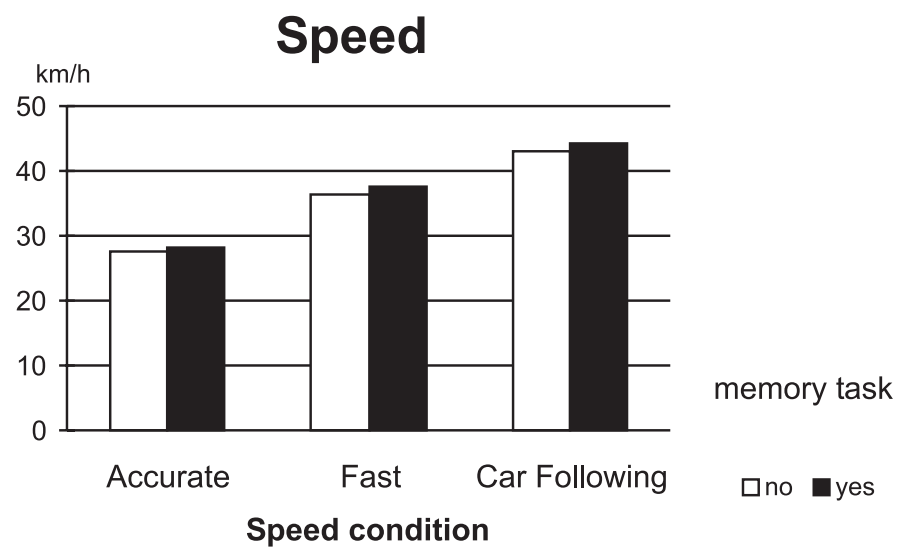

Fig. 1. Average driving speed across the experimental conditions. 


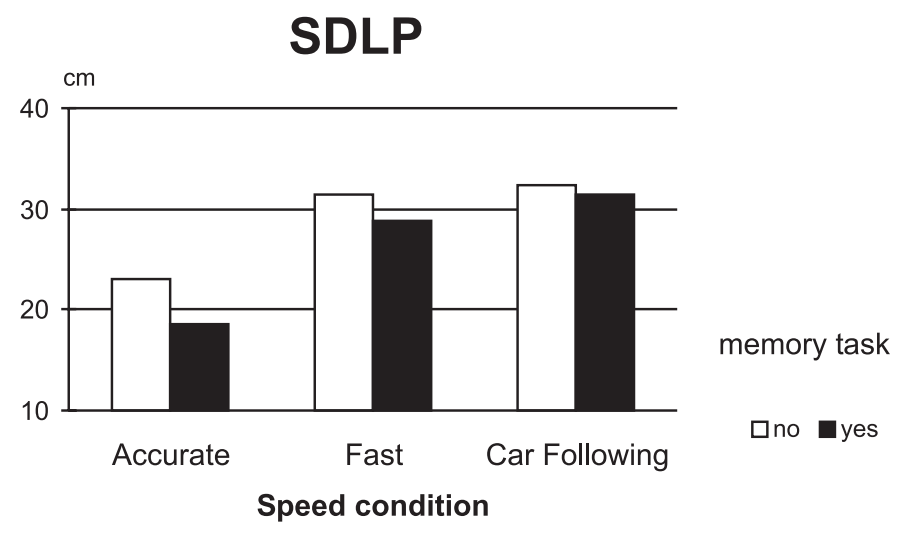

Fig. 2. Average SD lateral position across the experimental conditions.

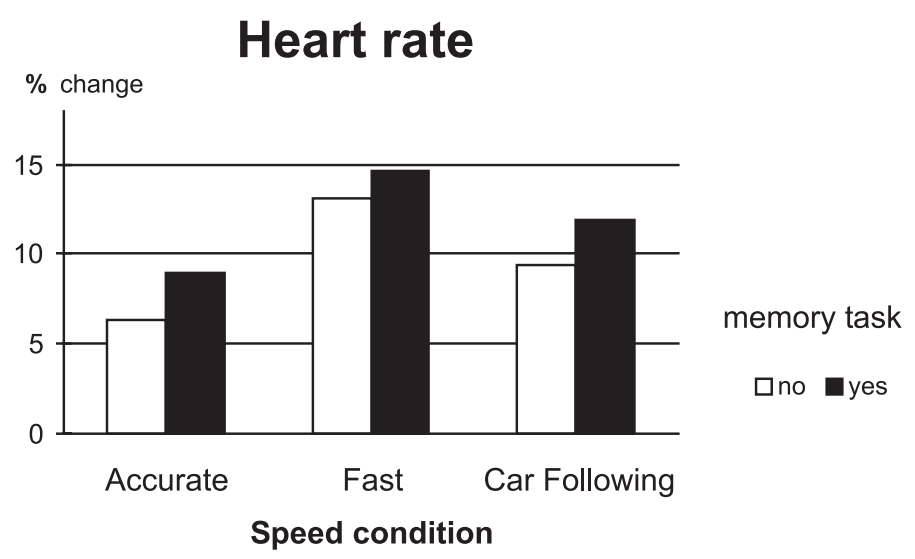

Fig. 3. Average SD steering wheel angle across the experimental conditions.

speed $(F(1,13)=.978 ; \mathrm{ns})$. For averaged $S D L P$ (see Fig. 2$)$, the main effect of speed instruction was significant (Hotelling's $T=1.29 ; P<.009$ ), but somewhat surprisingly, none of the pair-wise analyses (Fast vs Accurate, Fast vs Following) showed statistically significant effects, probably due to the relatively large standard deviations of SDLP. The main effect of speed for averaged SD steering wheel angle (see Fig. 3) was also significant (Hotelling's $T=2.73 ; P<.001$ ). Pair-wise analyses showed that this was due to the significant difference between the Fast and Accurate condition $(F(1,13)=4.78 ; P<.049)$; the difference between the Fast and Car Following condition was not significant $(F(1,13)=.72 ; \mathrm{ns})$. There was no significant effect of the memory task on $\operatorname{SD}$ Steer $(F(1,13)=1.91 ; \mathrm{ns})$.

\subsection{Memory task}

The percentages of participants that gave the correct location and length of the longest traffic jam in the list for each speed condition were as followed: Accurate: 71; Fast: 86; Car 
Following: 64. Thus, memory task performance was highest in the Fast condition and lowest in the Car Following condition, but the differences between conditions were not significant. McNemar's test of significance of changes showed no significant differences between the Fast and Accurate conditions $(P<.688)$, nor between the Fast and Car Following conditions $(P<.453)$.

\subsection{Mental effort}

Fig. 4 shows the averaged percentage increase in heart rate (in fact interbeat interval) relative to baseline: the higher the change, the more effort was invested; $10 \%$ change means that IBI decreased $10 \%(=$ increase in heart rate) compared to baseline. The multivariate test showed a significant main effect of speed conditions (Hotelling's $T=1.91 ; P<.002$ ). Pair-wise comparisons showed that heart rate in the Fast condition was significantly higher than in Accurate condition $(F(1,13)=24.600 ; P<.001)$, and higher than in Car Following condition $(F(1,13)=6.270 ; P<.027)$. The effect of the memory task on heart rate was also significant $(F(1,13)=4.66 ; P<.050)$ : when participants performed the memory task, their heart rate was increased compared to conditions when travel news could be ignored.

The decrease in heart rate variability (HRV), indicating an increase in mental effort, was highest for the Fast condition (see Fig. 5, higher bars indicates more mental effort). The main multivariate effect of speed condition on HRV approached significance (Hotelling's $T=.59 ; P<.07$ ). Pairwise analyses showed that the difference in HRV between the Fast condition and the Accurate condition approached significance $(F(1,13)=3.886 ; P<.07)$, while the difference between the Car Following condition and the Fast condition was significant $(F(1,13)=5,387 ; P<.037)$. The memory task had no significant effect on $\operatorname{HRV}(F=.404$; ns $)$.

\section{HRV}

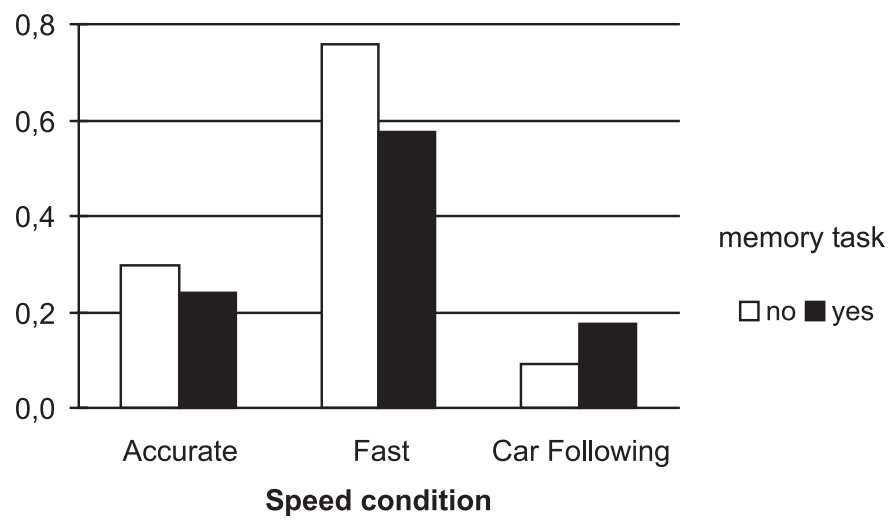

Fig. 4. Average percentage change in inter-beat interval between baseline and the conditions. Higher bars indicate higher heart rate in the experimental condition. 


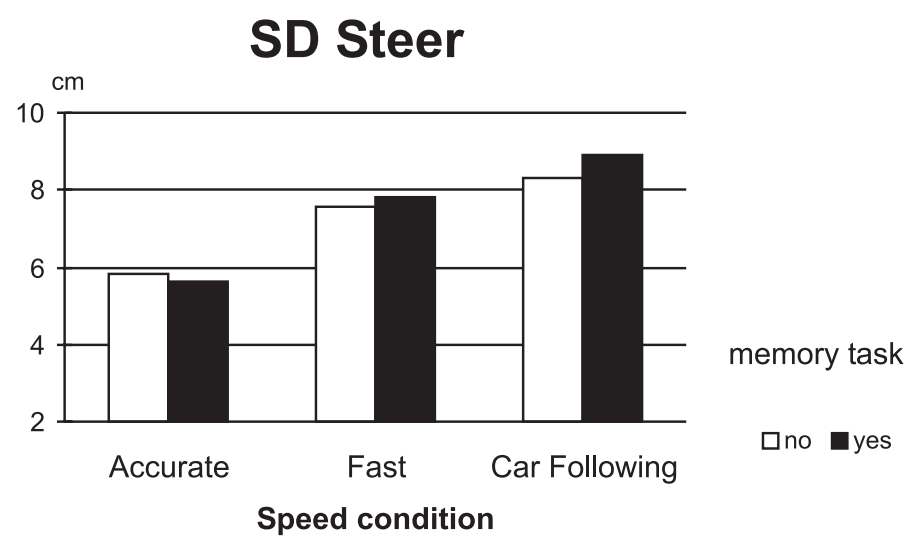

Fig. 5. Average change in heart rate variability between baseline and the conditions (see text for more information). Higher bars indicate more cognitive effort in the experimental condition.

\section{RSME}

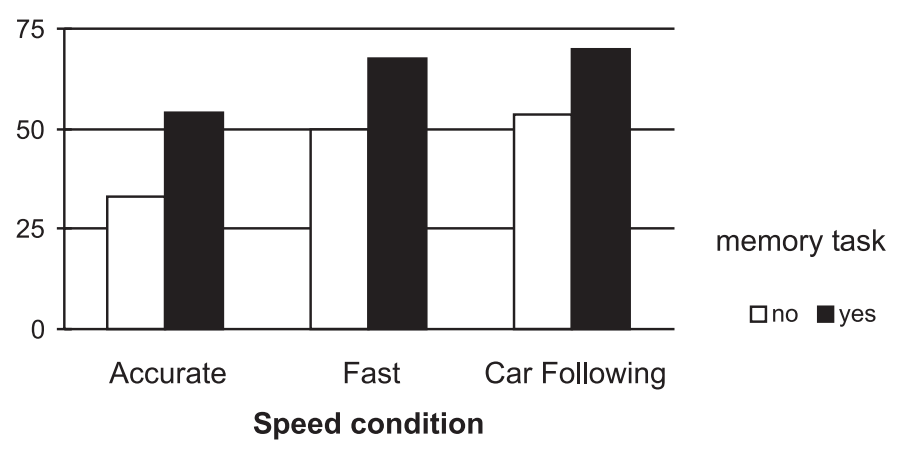

Fig. 6. Average self-reported mental effort across the experimental conditions. The maximum value of the scale is 150 .

\subsection{Self-reported effort}

Fig. 6 shows the averaged scores on the RSME; the multivariate effect of speed condition was significant (Hotelling's $T=2.44 ; P<.001$ ). Self-reported effort was significantly higher in Fast than in Accurate conditions $(F(1,13)=12.931 ; P<.003)$, but there was no significant difference between Fast and Car Following conditions $(F(1,13)=.447$; ns). The participants rated rides in which the memoroy task was performed as more demanding $(F(1,13)=36.475 ; P<.001)$.

\section{Discussion}

A main assumption of the behavioural adaptation model by Summala $(1996,1997)$ is that drivers try to keep available time or workload constant. An important way of doing so is adapting 
behaviour to the task demands: high task demands result in lower driving speed and/or less attention to additional tasks while driving; low task demands lead to increased driving speed, or more attention to additional tasks. The model does not provide specific predictions regarding the priorities which drivers assign to specific additional tasks. Hockey (1997) compensatory control model, however, predicts that drivers' priority is with the protection of the main task goal. In driving, the main task goal is to arrive safely at the planned destination. This implies, for example, that the additional task of processing route guidance information is more important to drivers that performing a working memory task unrelated to the driving task. A previous experiment indeed confirmed this prediction (Cnossen et al., 2000). Drivers were found to give primacy to the route information more than to performing an auditory memory task. They were also found to reduce their driving speed when route information was difficult to acquire or when traffic density was high, but not when performing a memory task.

Car drivers have often been found to reduce their driving speed in situations of high (primary) task demands. It has been assumed that this speed reduction serves to decrease task demands, but this relation between driving speed and mental effort has not been tested directly. The question that this driving simulator experiment tried to answer was therefore whether this speed reduction is indeed a strategy to reduce the task demands, perhaps to protect the main task goal. Participants were given different speed instructions: driving accurately, driving fast, or following a car driving fast. The prediction was that, all other things being equal, task demands should be higher in the Fast and the Car Following conditions than in the Accurate condition, due to higher driving speeds. Only if driving speed and task demands are inversely related, can speed reductions be interpreted as a way to reduce mental effort.

An additional objective of the study was to test the prediction of the behavioural adaptation model that low task demands are associated with better performance on a secondary task. Therefore, all speed conditions were driven with and without a memory task. From the general literature came the prediction that the memory task, not being relevant for the driving task, might be neglected in high task demand situations. The behavioural adaptation model predicts that because workload should remain constant, low task demands of the Accurate condition should be compensated for by better performance on the memory task. Furthermore, with task demands as the control variable, mental effort should be the same for all conditions.

The main reason for including the Accurate condition was to reduce driving speed of the participants. The instructions to the participants were indeed successful in inducing lower driving speeds in the Accurate condition than in the other conditions. However, SDLP did not show that participants actually drove more accurately in the Accurate condition. Obviously, an instruction to drive as accurately as possible should lead to more accurate lateral control. However, the SD steering wheel angle was lower in the Accurate condition. This indicates that participants needed less steering wheel movements to achieve the same level of lateral control. That is, drivers were steering more accurately in the Accurate condition than in the other conditions, where more and/ or larger steering corrections were needed to maintain the same lateral position (cf. Donges, 1978).

The high level of task performance in the Fast condition is striking. The task of the participants was a demanding one: not only did they have to drive fast, interact with other traffic, and monitor the auditory information for RG messages, but at the same time, they also had to process the travel news, remembering the location and length of the longest traffic jam in the list, replacing it by longer jams as they came along. It is therefore, quite remarkable that despite the high driving 
speed, $86 \%$ of the participants were able to name the correct location and length of the longest traffic jam at the end of the ride. This shows that even when driving fast, car drivers can process large amounts of information and perform additional tasks. In other words, even in these difficult situations, car drivers did not seem to suffer from 'driver overload'. However, this high level of performance had costs associated with it. Compared to the Accurate condition, HR and RSME showed significant increases in the Fast condition, while the differences in HRV approached significance.

Obviously, this is in contrast with the prediction from the behavioural adaptation model that mental effort should remain constant across conditions. Also, memory task performance was not better in the Accurate condition than in the other conditions. That is, drivers did not compensate for the lower task demand levels of the Accurate condition by paying more attention to the memory task, as the behavioural adaptation model predicts. Thus, the present study found no evidence for the notion that low task demands (in this study because of low driving speed) lead to an increase in secondary task performance, which seriously questions the validity of that assumption.

The results for the Car Following condition were not as straightforward as the results for the Fast and Accurate condition. Although driving speed in the Car Following condition was significantly higher than in the Fast condition, and heart rate was also significantly increased, participants did not report more mental effort in the Car Following condition. HRV even showed that participants invested significantly less mental effort in the Car Following than in the Fast condition. Post-hoc analyses showed no significant differences in HRV between the Car Following and the Accurate conditions $(F(1,13)=.255$; ns), although driving speeds in these conditions were markedly different. This is clearly in contrast to the general prediction that higher driving speeds are associated with more mental effort.

First, what might be the reason for the dissociation in the mental effort measures? The fact that heart rate was increased in the Car Following condition compared to the Fast condition indicates increased arousal, and perhaps increased anxiety about the task. The car to be followed drove faster than participants in the Fast condition, and participants may have been worried about losing it in traffic. Also, higher driving speeds might have induced more frequent physical activities (steering, shifting gear, etc.), increasing physical activity in the Car Following condition, which might have increased heart rate. HRV, on the other hand, is not sensitive to changes in physical effort during driving (Egelund, 1982). Generally speaking, HRV is specifically sensitive to cognitive effort, that is, manipulation of information in working memory (e.g., see Aasman et al., 1987; Wiethof, 1997). The data thus suggest that in the Car Following condition only little cognitive effort was invested.

An unintended aspect of the task structure might clarify this unexpected result. RG messages were presented auditorily in the Fast and Accurate condition, but not in the Car Following condition. Actually, RG messages were embedded within the travel news. This made the auditory information more important for the participants than we had intended. Participants had to monitor the travel news in all rides of the Fast and Accurate conditions for RG messages, and not only when they had to perform the memory task. In the Car Following condition, participants simply had to follow a car to know the way to drive. The important difference between the Car Following and other conditions, therefore, seems to be the easy access to RG information. Cues easily observable in the immediate external environment took away the need for demanding cognitive processing, and thus reduced the cognitive demands on the operator. 
In contrast, in the Fast and Accurate conditions, the need to monitor the auditory information was high. Actually, not only did the drivers have to remember and update the traffic queues when appropriate, but they also had to detect whether a message was a travel news message or a route guidance message. Furthermore, there was a need to synchronise the auditory route guidance message with the visual cues in the environment. The actual task for the participants therefore involved a significant amount of switching between different sources of information. Task switching has a strong association with cognitive effort and therefore with HRV. It is conceivable that this synchronisation of different types of information is especially strained when driving fast, as in the Fast condition. This would explain the difference between the Fast and Accurate condition. Unfortunately, HRV cannot be computed reliably on a messageto-message basis to test this directly, but this interpretation is supported by the Car Following condition, in which processing of the auditory information was not required. Despite higher driving speed, HRV showed that cognitive effort was significantly lower in the Car Following than in the Fast condition.

This interpretation would also explain the unexpected lack of effects of the memory task on HRV. HRV was expected to show an increase due to cognitive effort when participants had to remember the longest traffic jam. However, as the RG messages were embedded within the travel news, participants had to process the travel news in all rides of the Accurate and Fast conditions. Therefore, performing the memory task had no additional effects on HRV in the Fast and Accurate condition. In this context, it is noteworthy that memory task performance was lowest in the Car Following condition, indicating that participants indeed paid less attention to the travel news than in the other speed conditions. However, these data should be interpreted with some reservations, as the differences between speed conditions in memory task performance were not significant. Also, the high performance in the demanding Fast condition may indicate that the task is actually insensitive because of a ceiling effect. On the other hand, the memory task performance measure was dichotomous, and the number of participants may have been too small to be able to detect differences.

In car driving, the main task goal is to arrive at the planned destination. It is therefore essential for car drivers to know the route to drive, and this implies that in unfamiliar environments, route guidance information should not be missed. The present study showed that when RG information is embedded within other information, much effort is invested to extract that information. Especially in the conditions where participants were driving fast (Fast and Car Following), mental effort (RSME, HR) was high. More importantly, this effect on mental effort even existed in those conditions where travel news was presented but participants could ignore the information. This should have implications for the design of route guidance systems and other in-car information systems. Because driving is largely a visual task, researchers have suggested (e.g., Schraagen, 1993; Wierwille, 1993) that RG systems should not present their information visually, as this would further increase visual task demands. Instead, information should be given auditorily.

However, the present study showed that presenting information auditorily can also increase task demands substantially, especially when the auditory information is embedded within other auditory information. The Fast and Car Following conditions showed substantial interference, due to the primary task instruction (navigation messages) and secondary task information (travel news) both being auditory. This is in agreement with the multiple resource model by Wickens $(1984,1991)$, which assumes separate resources for two different codes of information (verbal and 
spatial) and for two different stages of information processing (perceptual/cognitive and response stages). This suggests that important information should not be presented in the same modality. In a previous study (Cnossen et al., 2000), however, a memory task was presented auditorily while route information was presented visually as a paper map. The multiple resources theory predicts that this should not interfere. However, substantial interference was observed between the two information sources: memory task performance decreased considerably when participants were driving with a map. Presenting information in different modalities, therefore, does not seem to suffice to prevent interference. It was concluded that the priority of the information was probably more important for interference than the modality of the information presented: more important information receives more attention. Drivers focus more on navigation information than on an artificial memory task, because route information is more central to the driving task. This is indeed a central tenet in the general literature on complex dynamic tasks (Bainbridge, 1974; Hockey, 1993, 1997): tasks are prioritised with respect to the main task goal.

Unfortunately, the relation between task demands and driving speed could not be studied as directly in the present experiment as was hoped, as a few factors were confounded. A follow-up study rectifying this problem would, therefore, be of great interest. An interesting research question would be whether modality of the information presented is the determining factor in task interference or whether priority of information is more important.

In summary, as the lack of HRV effects in the Car Following condition showed, high driving speed alone does not necessarily increase cognitive effort. Only when high driving speed is associated with performing a cognitively demanding task, is cognitive effort increased. From the results of the Accurate and Fast conditions, it appears that car drivers may indeed reduce their driving speed to decrease task demands, and by doing so, decrease the necessary mental effort. However, the present study found no evidence for the assumption that low task demands increase secondary task performance. On the contrary, task behaviour appears more strategic. Drivers prioritise their tasks with respect to the main task goal, which is to arrive safely at the destination. Also, not all increases in task demands are automatically compensated for by increases in mental effort, but effort investment is strategic and motivational. It thus appears, that the behavioural adaptation model could benefit from including more general psychological mechanisms to explain driver behaviour.

The main issue in previous traffic behaviour research concerning subsidiary tasks was that drivers might become overloaded or distracted by additional information. Our results show that car drivers can achieve a high level of task performance when performing a number of tasks concurrently, even in difficult driving situations. However, drivers will try to avoid the high costs associated with this by either skipping the less important subtasks, or by adapting their driving behaviour. The main concern should, therefore, be with where these adaptive responses are not possible, either because of high traffic densities, high speeds, or because of strict time schedules of the professional driver.

\section{References}

Aasman, J., Mulder, G., \& Mulder, L. J. M. (1987). Operator effort and the measurement of heart-rate variability. Human Factors, 29, 161-170. 
Alm, H., \& Nilsson, L. (1995). The effects of a mobile telephone task on driver behaviour in a car following situation. Accident Analysis and Prevention, 27, 707-715.

Bainbridge, L. (1974). Problems in the assessment of mental load. Le Travail Human, 37, 279-302.

Brookhuis, K. A., De Vries, G., \& De Waard, D. (1991). The effects of mobile telephoning on driving performance. Accident Analysis and Prevention, 23, 309-316.

Brown, I. D. (1962). Measuring the 'spare mental capacity' of car drivers by a subsidiary auditory task. Ergonomics, 5, 247-250.

Brown, I. D., \& Poulton, E. C. (1961). Measuring the spare 'mental capacity' of car drivers by a subsidiary task. Ergonomics, 4, 35-40.

Cnossen, F., Meijman, T.F., \& Rothengatter, T. (2000). Adaptive strategy changes as a function of task demands: a study in car drivers (Submitted).

Crundall, D. E., \& Underwood, G. (1998). Effects of experience and processing demands on visual information acquisition in drivers. Ergonomics, 41, 448-458.

Crundall, D., Underwood, G., \& Chapman, P. (1999). Driving experience and the functional field of view. Perception, $28,1075-1087$.

De Waard, D. (1991). Driving behaviour on a high-accident-rate motorway in the Netherlands. In C. Weikert, K.A. Brookhuis, \& S. Ovinius (Eds.), Man in complex systems. Proceedings of the Human Factors Society Annual Meeting, (Work Science Bulletin 7) (pp. 113-123). Lund, Sweden: Work Science Division, Department of Psychology, Lund University.

Dingus, T. A., Hulse, M. C., Mollenhauer, M. A., Fleischman, R. N., McGehee, D. V., \& Manakkal, N. (1997). Effects of age, system experience, and navigation technique on driving with an advanced traveler information system. Human Factors, 39, 177-199.

Donges, E. (1978). A two-level model of driver steering behavior. Human Factors, 20, 691-707.

Egelund, N. (1982). Spectral analysis of heart rate variability as an indicator of driver fatigue. Ergonomics, 25, 663-672.

Fairclough, S.H., Ashby, M.C., \& Parkes, A.M. (1993). In-vehicle displays, visual workload and usability evaluation. In A.G. Gale, I.D. Brown, C.M. Haslegrave, H.W. Kruysse, \& S.P. Taylor, Vision in vehicles iv (pp. 245-254). Amsterdam: Elsevier.

Fuller, R. G. C. (1981). Determinants of time headway adopted by truck drivers. Ergonomics, 24, 463-474.

Hancock, P. A., \& Caird, J. K. (1993). Experimental evaluation of a model of mental workload. Human Factors, 35, 413-429.

Harms, L. (1991). Variation in drivers' cognitive load. Effects of driving through village areas and rural junctions. Ergonomics, 34, 151-160.

Hockey, G.R.J. (1993). Cognitive-energetical control mechanisms in the management of work demands and psychological health. In A. Baddeley, \& L. Weiskrantz (Eds.), Attention: selection, wareness, and control (pp. 328345). Oxford: Oxford University Press.

Hockey, G. R. J. (1997). Compensatory control in the regulation of human performance under stress and high workload: a cognitive-energetical approach. Biological Psychology, 45, 73-93.

Hockey, G. R. J., Wastell, D. G., \& Sauer, J. (1998). Effects of sleep deprivation and user interface on complex performance: a multilevel analysis of compensatory control. Human Factors, 40, 233-253.

Landsdown, T.C. (1997). Visual allocation and the availability of driver information. In J.A. Rothengatter, \& E. Carbonell Vaya (Eds.), Traffic and transport psychology: theory and application (pp. 215-223). Oxford: Pergamon.

Michon, J.A. (1985). A critical view of driver behavior models: what do we know, what should we do? In L. Evans, \& R.C. Schwing (Eds.), Human behavior and traffic safety (pp. 485-524). New York: Plenum Press.

Mulder, G., \& Mulder, L. J. M. (1990). Information processing and cardiovascular control. Psychophysiology, 18, 392-402.

Mulder, L.J.M., Van Dellen, H.J., Van der Meulen, P., \& Opheikens, B. (1988). CARSPAN, A spectral analysis program for cardiovascular time series. In F.J. Maarse, L.J.M. Mulder, W. Sjouw, \& A. Akkerman (Eds.), Computers in psychology: methods, instrumentation and psychodiagnostics (pp. 30-38). Lisse, The Netherlands: Swets \& Zeitlinger.

Näätänen, R., \& Summula, H. (1976). Road-user behaviour and traffic accidents. Amsterdam: North-Holland.

Noy, Y.I. (1989). Intelligent route guidance: will the new horse be as good as the old? Proceedings of the vehicle navigation and information system conference (VNIS) (pp. 49-55). Toronto. 
Pohlmann, S., \& Traenkle, U. (1994). Orientation in road traffic. Age-related differences using an in-vehicle navigation system and a conventional map. Accident Analysis and Prevention, 26, 689-702.

Ranney, T. A. (1994). Models of driving behavior: a review of their evolution. Accident Analysis and Prevention, 26, 733-750.

Schraagen, J.M.C. (1993). Information presentation in in-car navigation systems. In A.M. Parkes, \& S. Franzèn (Eds.), Driving future vehicles (pp. 171-185). London: Taylor \& Francis.

Summula, H. (1985). Modelling driver behavior: a pessimistic prediction? In L. Evans, \& R.C. Schwing (Eds.), Human behavior and traffic safety (pp. 43-61). New York: Plenum Press.

Summala, H. (1996). Accident risk and driver behaviour. Safety Science, 22, 103-117.

Summala, H. (1997). Hierarchical model of behavioural adaptation and traffic accidents. In J.A. Rothengatter, \& E. Carbonell Vaya (Eds.), Traffic and transport psychology: theory and application (pp. 41-52). Oxford: Pergamon.

Taylor, D. H. (1964). Drivers' galvanic skin response and the risk of accident. Ergonomics, 7, 253-262.

Van der Hulst, M., Meijman, T.F., \& Rothengatter, J.A. (2000). Prolonged driving: maintaining task set under fatigue (Submitted).

Van Roon, A.M. (1998). Short-term cardiovascular effects of mental tasks. Physiology, experiments and computer simulations. Doctoral thesis, University of Groningen.

Van Wolffelaar, P.C., \& Van Winsum, W. (1995). Traffic modelling and driving simulation an integrated approach. In DSC '95. Driving simulation conference, Conference proceedings (pp. 236-244). Toulouse: Teknea.

Verwey, W.B. (1993). How can we prevent overload of the driver? In A.M. Parkes \& S. Franzèn (Eds.), Driving future vehicles (pp. 235-244). London: Taylor \& Francis.

Verwey, W. B., \& Veltman, H. A. (1996). Detecting short periods of elevated workload: a comparison of nine workload assessment techniques. Journal of Experimental Psychology: Applied, 2, 270-285.

Wickens, C.D. (1984). Processing resources in attention. In R. Parasuraman, \& D.R. Davies (Eds.), Varieties of attention (pp 63-102). New York: Academic Press.

Wickens, C.D. (1991). Processing resources and attention. In D.L. Damos (Ed.), Multiple-task performance (pp. 3-34). London: Taylor \& Francis.

Wilde, G. J. S. (1982). The theory of risk homeostasis: implications for safety and health. Risk analysis, 2, $209-225$.

Wilson, G.F., \& Eggemeier, F.T. (1991). Physiological assessment of workload in multi-task environments. In D.L. Damos (Ed.), Multiple-task performance (pp. 329-360). London: Taylor \& Francis.

Wierwille, W. W. (1993). Demands on driver resources associated with introducing advanced technology into the vehicle. Transportation Research-C, 1, 133-142.

Wiethof, M. (1997). Task analysis is heart work. The investigation of heart rate variability: a tool for task analysis in cognitive work. Doctoral thesis. Delft: Delft University Press.

Zijlstra, F. R. H. (1993). Efficiency in work behaviour: a design approach for modern tools. Delft: Delft University Press (Doctoral thesis). 